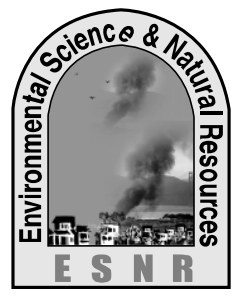

J. Environ. Sci. \& Natural Resources, 6(1): 21 - 32 , 2013

ISSN 1999-7361

\title{
Biodiversity Status and its Management at Ramsagar National Park at Dinajpur in Bangladeh
}

\author{
R. H. Rimi, F. Rahman, and M. B. Latif \\ Department of Environmental Science and Resource Management \\ Mawlana Bhashani Science and Technology University, Tangail-1902
}

\begin{abstract}
According to this study a heterogeneous species assemblage of 393 species including 272 plants, 24 waterfowl and birds, 14 miscellaneous fauna and 84 species of fish was found at Ramsagar National Park. Among the entire plant types weed was found to be most abundant $(36.76 \%$ ), followed by the medicinal plants $27.57 \%$, ornamental plants $15.07 \%$, timber plant species $10.29 \%$, fruit plants $8.46 \%$, aquatic plants $6.62 \%$, cultivated crops $6.25 \%$, fodder plant $2.21 \%$, spice plants $2.21 \%$, and palm tree $1.84 \%$. All kinds of plants were very common in the study area. In case of fishes, $13.10 \%$ was cultivated and $63.10 \%$ was small indigenous but $23.81 \%$ of small indigenous fishes were threatened. At the study area, 9 species of bank birds (37.50\%), 4 species of prey birds (16.67\%), and 11 species of waterfowl (45.83\%) were found. Among the bank birds, 3 were very common and 4 were few. Among the 4 prey birds 3 species were very few and 1 species was few. In case of waterfowls, 5 species were few, 4 were common, and 1 species was very few. Seven species of miscellaneous faunal species were common, 1 very common, 3 few, and 3 were threatened. Contribution of biodiversity to the local people was mainly for food, although they used plants for furniture, fuel wood, wood, medicine, domestication etc. There was no management plan in the RNP but they had an annual work plan. Only 8 staffs were found for maintaining the Park which could not be adequate for proper management.
\end{abstract}

Key words: Biodiversity, Contribution, Management.

\section{Introduction}

Ramsagar is a historical National Park under the Forest Division of Dinajpur district in Bangladesh (Banglapedia, 2006). Due to anthropogenic pressures biodiversity of forests are decreasing rapidly. In order to conserve biodiversity, it is required to have precise data of current status of it. Taking into account of this requisite, Rahman (1989) assessed fresh water fisheries of Bangladesh; Islam (2006) studied the biodiversity of Jamuna River and its surrounding area. The co-management of protected areas in Bangladesh was studied by Huda (2008), while, the Forest Department's institutional organization and capacity to manage the protected area system of Bangladesh was investigated by IRG (2004). Islam (2001) studied on the status of Bhawal National Park exploring whether or not the objectives of protected areas have being met but no such studies was found on biodiversity of RNP which are biologically and environmentally important as well.

Therefore, this study was conducted at RNP with the following objectives: a) to assess diversity of species and thereby the biodiversity composition of the study area, b) to evaluate the contribution of biodiversity to the local community, and c) to depict the existing management structure of RNP.

\section{Materials and methods}

\section{Study Area}

The study area is located within the Sadar Upazila at approximately between the latitudes of $25^{\circ} 44^{\prime}$ and $25^{\circ} 33^{\prime} \mathrm{N}$ and between the longitudes of $88^{\circ} 30^{\prime}$ and $88^{\circ} 44^{\prime} \mathrm{E}$ at Dinajpur district. It is located $8.0 \mathrm{~km}$ south from Dinajpur town with Paikpara and Tajpur Mouza. It is about $50 \mathrm{~km}$ from Syedpur airport and $420 \mathrm{~km}$ by road from Dhaka (Banglapedia, 2006). Earlier the RNP had an area of 146.44 acres but now the total land area has reduced to 68.54 acres which is categorized as a reserved forest under the Gazette notification of 24 December 1974.

\section{Data collection}

The biodiversity status was recorded through identifying and recording all species present at RNP. During the field survey, observations were made on the habitats, leaves, flowers, fruits, abundance of the plant etc. An attempt was made to identify the plants and animals on the spot. Those plants that could not be identified were preserved in herbarium with flowers and fruits for identification with help of the taxonomist. Different types of standard taxonomic books have been consulted for collection of scientific names and the relevant information (Rashid, 1990). Prior to field visit, the detailed map of RNP was consulted. A questionnaire survey and Key Informant Interview (KII) were conducted for the study. For the purpose of survey, the simple random sampling was used and the sample size was 100 . Key informants included Divisional Forest Officer, Dinajpur Divisional Forest Office and Forest Officer, RNP to ascertain species diversity of plants, animals, birds, waterfowl and fishes in the study area and its surrounding areas at Dinajpur. 


\section{Results and discussion}

According to this study, the National Park was found to be a home of total 104 animal species representing 95 genera and 46 families and 272 plants species representing 237 genera and 132 families respectively. Plant types included timber, fruit, medicinal, fodder, palm, spices, ornamental, aquatic plants, cultivated crops and weed species group whereas animals could be categorized into cultivated fish, small indigenous fish, threatened small indigenous fish, bank bird, bird of prey, and waterfowls group. The plants species of timber were 28 , fruit 19 , medicinal 75 , fodder 17 , palm 06 , spices 05 , ornamental 06 , aquatic plants 41 , cultivated crops 18 and weed species 57 . The cultivated fish group had 11 species, small indigenous fish 53, threatened small indigenous fish 20, bank birds 9 , bird of prey 4 and waterfowl 11 species.

\section{Plant Biodiversity}

According to the local people plant biodiversity in the study area was not decreasing because of the numbers of plants were increasing by the program of social forestry under Divisional Forest Office (DFO), Dinajpur. In the National Park 18.22 and 2 ha land was used for indigenous and exotic plantations respectively. Table 1 , Table 2, Table 3 , and Table 4 consequently show the biodiversity of timber plants, fruit plants, medicinal plants and cultivated crops species observed in the study area which were classified into tree, shrub, herb and climber plant types.

Table 1. Biodiversity of timber plant species observed in the study area

\begin{tabular}{|l||l||l||l||}
\hline \multicolumn{1}{|c||}{ Local name } & \multicolumn{1}{c|}{ Family } & \multicolumn{1}{c||}{ Plant type } \\
\hline Eucalyptus & Myrtaceae & Eucalyptus camaldulensis & Tree \\
\hline \hline Khudijam & Myrtaceae & Eugenia fruticosa & Tree \\
\hline Akashmoni & Mimosaceae & Acacia auriculiformis & Tree \\
\hline \hline Rain tree & Mimosaceae & Albizia saman & Tree \\
\hline \hline Sada koroi & Mimosaceae & Albizia procera & Tree \\
\hline Kala koroi & Mimosaceae & Albizia lebbeck & Tree \\
\hline Mangium & Mimosaceae & Acacia mangium & Tree \\
\hline \hline Raj koroi & Mimosaceae & Albizia richardiana & Tree \\
\hline \hline Lohakat & Mimosaceae & Xylia dolabiformis & Tree \\
\hline \hline Sissoo & Papilionaceae & Dalbergia sissoo & Tree \\
\hline \hline Pitali & Euphorbiaceae & Trewia polycarpa & Tree \\
\hline \hline Sinduri & Euphorbiaceae & Mallotus philippinesis & Tree \\
\hline \hline Jarul & Lythraceae & Lagerstroemia speciosa & Tree \\
\hline \hline Mehogoni & Meliaceae & Swietenia macrophylla & Tree \\
\hline \hline Kadam & Rubiaceae & Anthocephalus chinensis & Tree \\
\hline \hline Sheora & Urtieaceae & Sterblus asper & Tree \\
\hline \hline Sonalu & & Cassia fistula & Tree \\
\hline \hline Simul & Bombaceae & Bombax ceiba & Tree \\
\hline \hline Bot & Moraceae & Ficus bengalensis & Tree \\
\hline \hline Pakur & Moraceae & Ficus comosa & Tree \\
\hline \hline Dumur & Moraceae & Ficus carica & Tree \\
\hline \hline Bas & Gramineae & Bambusa aurundinaceae & Tree \\
\hline Debdaru & Annonaceae & Polyalthia longifolia & Tree \\
\hline Sal & Dipterocarpaceae & Shorea robusta & Tree \\
\hline \hline Telsur & Dipterocarpaceae & Hopea odorata & Tree \\
\hline \hline Toon & Meliaceae & Cedrela toona & Tree \\
\hline \hline Shegun & Verbenaceae & Tectona grandis & Tree \\
\hline \hline Gamari & Gerbenaceae & Gmelina arborea & \\
\hline & & & \\
\hline
\end{tabular}


Table 2. Biodiversity of fruit plant species identified in the study area

\begin{tabular}{|l|l|l|l||}
\hline \multicolumn{1}{|c|}{ Local name } & \multicolumn{1}{c|}{ Family } & \multicolumn{1}{c||}{ Genus Species } & \multicolumn{1}{c||}{ Plant type } \\
\hline \hline Aam & Anacardiaceae & Mangifera indica & Tree \\
\hline \hline Amra & Anacardiaceae & Spondias pinnata & Tree \\
\hline \hline Kathal & Moraceae & Artocarpus heterophyllus & Tree \\
\hline \hline Dewa & Moraceae & Artocarpus lakoocha & Tree \\
\hline \hline Peyara & Myrtaceae & Psidium guajava & Tree \\
\hline Jam & Myrtaceae & Syzgium cumini & Tree \\
\hline \hline Jamrul & Myrtaceae & Syzgium samarangense & Tree \\
\hline \hline Boroi & Rhamnaceae & Zizyphus mauritiana & Tree \\
\hline \hline Chalta & Dilleniaceae & Dillenia indica & Tree \\
\hline Jambura & Rutaceae & Citrus grandis & Tree \\
\hline \hline Bel & Rutaceae & Aegle mermelos & Tree \\
\hline \hline Kola & Musaceae & Musa sapientum & Herb \\
\hline \hline Ata & Annonaceae & Annona reticulate & Tree \\
\hline \hline Sarifa ata & Annonaceae & Annona squamosa & Tree \\
\hline \hline Kamranga & Averrhoaceae & Averrhoa carambola & Tree \\
\hline \hline Tetul & Caesalpinieae & Tamarindus indica & Tree \\
\hline Jalpai & Elaeocarpaceae & Elaeocarpus robustus & Tree \\
\hline \hline Amloki & Euphorbiaceae & Phyllanthus embelica & Tree \\
\hline \hline Litchi & Sapindaceae & Litchi chinensis & Tree \\
\hline
\end{tabular}

Table 3. Biodiversity of medicinal plant species observed in the study area

\begin{tabular}{|c|c|c|c|}
\hline$\overline{\text { Local name }}$ & $\overline{\bar{F} \text { Family }}$ & Genus Species & Plant type \\
\hline Basok & Acanthaceae & Adhatoda vasica & Shrub \\
\hline Kalomegh & Acanthaceae & Andrographis paniculata & Shrub \\
\hline Katanoty & Amaranthaceae & Amaranthus spinosus & Herb \\
\hline Vehla & Anacardiaceae & Semecarpus anacardium & Tree \\
\hline Amloki & Annonaceae & Allamonda catharitca & Shrub \\
\hline Nayantara & Apocynaceae & Vinca rosea & $\begin{array}{l}\text { Shrub } \\
\end{array}$ \\
\hline Nayantara (Sada) & Apocynaceae & Vinca alba & Shrub \\
\hline Kolkeyphul & Apocynaceae & Thevetia nerifolia & Tree \\
\hline Sarpogandha & Apocynaceae & Rauwolfia serpentine & Shrub \\
\hline Boch & Araceae & Acarus calamus & Herb \\
\hline Ishwarmul & Aristolochiaceae & Aristolochia indica & Climber \\
\hline Anantamul & Asclepiadaceae & Hemidesmus indica & Herb \\
\hline Tecoma & Bignoniaceae & Tecoma stans chelonioids & Shrub \\
\hline Parul & Bignoniaceae & Stereospermum & Tree \\
\hline Banhalud & Bixaceae & Bixa orellana & Shrub \\
\hline Mohneem & Burseraceae & Allananthes excels & Tree \\
\hline Kalokesunda & Caesalpinieae & Cassia sophera & Shrub \\
\hline Choto Kalokesunda & Caesalpinieae & Cassia tora & Shrub \\
\hline Kesuti & Compositae & Eclipta alba & Herb \\
\hline Dadmardon & Caesalpinieae & Cassia alata & Shrub \\
\hline Ashoke & Caesalpinieae & Saraca indica & Tree \\
\hline Bohera & Combretaceae & Terminalia belerica & Tree \\
\hline Horitoki & Combretaceae & Terminalia hebula & Tree \\
\hline Mohavinghoraj & Compositae & Wedilia calendulacea & Herb \\
\hline Bhui kumra & Convolvuaceae & Ipomoea digitata & Shrub \\
\hline Sharnalata & Convolvuaceae & Cuscuta reflexa & Climber \\
\hline
\end{tabular}




\begin{tabular}{|c|c|c|c|}
\hline Local name & Family & Genus Species & Plant type \\
\hline Pathor kuchi & Crassulaceae & Bryophullum calycium & Herb \\
\hline Mateaalu & Dioscoriaceae & Dioscorea alata & Climber \\
\hline Sinduri & Euphorbiaceae & Mallotus philippinensis & Tree \\
\hline Bishkhagor & Euphorbiaceae & Croton tiglium & Tree \\
\hline Verenda & Euphorbiaceae & Ricinus communis & Shrub \\
\hline Bissatu & Euphorbiaceae & \begin{tabular}{|l|} 
Tragia involuerata \\
\end{tabular} & \begin{tabular}{|l|} 
Climber \\
\end{tabular} \\
\hline Lemon ghash & Gramineae & Andropogon citrates & Herb \\
\hline Kash & Gramineae & Saccharum spontaneum & Herb \\
\hline Nageshwarchapa & Gramineae & Mesuaferrea & Shrub \\
\hline Shetodrone & Labiatae & Leucas aspera & Herb \\
\hline Kalotulsi & Labiatae & Ocimum sanctum & Shrub \\
\hline Ghritukumari & Liliaceae & Aloe barbadensis & Herb \\
\hline Shatamuli & Liliaceae & Asparagus sprengeri & Climber \\
\hline Agniswar & Liliaceae & Cardyline terminalis & Shrub \\
\hline Mehedi & Lythraceae & Lawsonia mermis & Shrub \\
\hline Neem & Meliaceae & Azadirachta indica & Tree \\
\hline Pitraj & Meliaceae & Amoora rohituka & Tree \\
\hline $\begin{array}{l}\text { Lazzabati } \\
\end{array}$ & Mimosaceae & Mimosa pudica & Herb \\
\hline Gila & Papilionaceae & Entada scandens & \begin{tabular}{|l} 
Climber \\
\end{tabular} \\
\hline Palash & Papilionaceae & Butea monosperma & Tree \\
\hline Roktachandon & Papilionaceae & Pterocarpus santalinus & Tree \\
\hline Pipul & Piperaceae & Piper longum & Climber \\
\hline Bishlong & Rubiaceae & Randia dumetorum & Shrub \\
\hline Pindal & Rubiaceae & Randia uliginosa & Shrub \\
\hline Mahua & Sapotaceae & Madhuca longifolia & Tree \\
\hline Bakul & Sapotaceae & Mimusops elengi & Tree \\
\hline Ulatkambol & Sterculiaceae & Abroma augusta & Shrub \\
\hline Agar & Thymelaceae & Aquiliaria nudiflora & Shrub \\
\hline Harjora & Vitaceae & Vitis quadrangularis & Tree \\
\hline Gulancha & Vitaceae & Tinospora cordifolia & Climber \\
\hline Nishinda & Verbanaceae & Vitex negundo & Climber \\
\hline Dholanchapa & Zingiberaceae & Hedychium coronarium & Shrub \\
\hline Banada & Zingiberaceae & Zingiber spectabile & Herb \\
\hline Chatim & Apocynaceae & Alstonia macrophylla & Tree \\
\hline Arjun & Combretaceae & Terminalia arjuna & Tree \\
\hline Akande & Asclepiadaceae & Calotropic procera & Tree \\
\hline Bishjarul & Acanthaceae & Justicia gendarussa & Shrub \\
\hline Goraneem & Meliaceae & Melia sempervirens & Tree \\
\hline Dhutora & Solanaceae & \begin{tabular}{|l} 
Datura metel \\
\end{tabular} & Shrub \\
\hline Chanchi & Amaranthaceae & | Alternanthera sessilis & Herb \\
\hline Hatisur & Boraginaceae & Heliotropium indicum & Herb \\
\hline Mutha & Cyperaceae & Cyperus alternifolium & Grass \\
\hline Muthavadhail & Cyperaceae & Cyperus rotundus & Grass \\
\hline Dol & Gramineae & Hygrorhiza aristata & Herb \\
\hline Tulsi & Labiatae & Ocimum sanctum & Herb \\
\hline Amrul & Oxalidaceae & Oxalis corniculata & Herb \\
\hline Thankuni & Umbelliferae & Hydrocotyle asiatica & Herb \\
\hline Bontamak & Solanaceae & Nicotiana plumbaginifolia & Herb \\
\hline Bonbegun & $\begin{array}{l}\text { Solanaceae } \\
\end{array}$ & Solanum fexox & $\begin{array}{l}\text { Shrub } \\
\end{array}$ \\
\hline
\end{tabular}


Table 4. Biodiversity of cultivated crops observed in the study area

\begin{tabular}{|l|l|l||l||}
\hline \multicolumn{1}{|c|}{ Common name } & \multicolumn{1}{c|}{ Gamily } & \multicolumn{1}{c|}{ Genus Species } \\
\hline \hline Rice & Gramineae & Oryza sativa & Herb \\
\hline \hline Wheat & Gramineae & Triticum aestivum \\
\hline \hline Maize & Gramineae & Zea mays & Herb \\
\hline \hline Sugarcane & Gramineae & Saccharum officinarum & Herb \\
\hline \hline Potato & Solanaceae & Solanum tuberosum & Herb \\
\hline \hline Brinjal & Solanaceae & Solanum melongena & Herb \\
\hline \hline Mustard & Cruciferae & Brassica campestris & Shrub \\
\hline \hline Black gram & Leguminosae & Latha mungo & Herb \\
\hline \hline Grass pea & Leguminosae & Lens culinaris & Herb \\
\hline \hline Lentil & Leguminosae & Vigna radius & Herb \\
\hline \hline Green gram & Leguminosae & Corchorus capsularis & Herb \\
\hline \hline Deshi jute & Tiliaceae & Corchorus olitorius & Herb \\
\hline \hline Tosa jute & Tiliaceae & Hibiscus subdariffa & Herb \\
\hline \hline Mesta jute & Malvaceae & Cajanus cajan & Herb \\
\hline \hline Arhar & Papilionaceae & Curcuma longa & Shrub \\
\hline \hline Turmeric & Zingiberaceae & Lagenaria siceraria & Herb \\
\hline \hline Bottle gourd & Cucurbitaceae & Herb \\
\hline \hline
\end{tabular}

Different ornamental plants, aquatic plants and weed species found at RNP are show in the Tables
5, 6, and 7. All the plants were classified into tree, shrub, herb and climber plant types.

Table 5. Biodiversity of ornamental plant species observed in the study area

\begin{tabular}{|l|l|l|l||}
\hline \multicolumn{1}{|c|}{ Local name } & \multicolumn{1}{c|}{ Family } & \multicolumn{1}{c|}{ Genus Species } & \multicolumn{1}{c||}{ Plant type } \\
\hline \hline Kathalichapa & Annonaceae & Artobotyris odoratissimus & Shrub \\
\hline \hline Tagor & Apocynaceae & Tabernaemontana odoratissimu & Shrub \\
\hline \hline Kolky & Apocynaceae & Thevetia neriiifolia & Tree \\
\hline \hline Malotilota & Apocynaceae & Aganosma caryophylla & Climber \\
\hline \hline Christmas tree & Araucariaceae & Arucarid cookie & Tree \\
\hline \hline Radhachura & Caesalpinieae & Caesalpinia pulcherrima & Tree \\
\hline \hline Kanchan & Caesalpinieae & Bauhinia purpurea & Tree \\
\hline \hline Krishnachura & Caesalpinieae & Delonix regia & Tree \\
\hline \hline Chandromollika & Compositae & Chrysanthemum segetum & Herb \\
\hline \hline Dahlia & Compositae & Dahlia imperialis & Herb \\
\hline \hline Ghada (African) & Compositae & Tagetes erecta & Herb \\
\hline \hline Ghada (French) & Compositae & Tagetes patula & Herb \\
\hline \hline Juniper & Cupressaceae & Juniperus prostate & Tree \\
\hline \hline Patromonjuri (red) & Euphorbiaceae & Euphorbia pulcherrima & Shrub \\
\hline \hline Patromonjuri (white) & Euphorbiaceae & Euphorbia sp. & Shrub \\
\hline \hline Silver fern & Filiaceae & Dryopteris extersa & Herb \\
\hline \hline Fern & Filiaceae & Ptetis sp. & Herb \\
\hline \hline Degar plant & Liliaceae & Yucca gloriosa & Shrub \\
\hline \hline Magnolia & Magnoliaceae & Magnolia alba & Shrub \\
\hline \hline Jhumkojoba & Malvaceae & Hibiscus schizopetalus & Shrub \\
\hline \hline Lankajoba & Malvaceae & Hibiscus sylvestris & Shrub \\
\hline \hline Joba & Malvaceae & Hibiscus rosasinensis & Shrub \\
\hline \hline Sthalpadda & Malvaceae & Hibiscus mutabilis & Shrub \\
\hline \hline Panthopathop & Musaceae & Ravenala madagascari \\
\hline \hline
\end{tabular}




\begin{tabular}{|c|c|c|c|}
\hline Local name & Family & Genus Species & Plant type \\
\hline Bottle brush & Myrtaceae & Callistemon linearis & Tree \\
\hline Lotabot & Moraceae & Fiscus pumila & Climber \\
\hline Bougainvillea & Nyctaginaceae & Bougainvillea grabra & Climber \\
\hline Kanakchapa & Ochanaceae & Ochna squarrrosa & Shrub \\
\hline Bely & Oleaceae & Jasminum sambac & Shrub \\
\hline Shefali & Oleaceae & Nyctanthes arbotristis & Shrub \\
\hline Thuja & Pinaceae & Thuja orientalis & Shrub \\
\hline Golap & Rosaceae & Rosa sp. & Shrub \\
\hline Sada rangan & Rubiaceae & Ixora arborea & Shrub \\
\hline Lal rangan & Rubiaceae & Ixora coccinea & Shrub \\
\hline Gandhoraj & Rubiaceae & Gardenia jasminoides & Shrub \\
\hline Musanda (white) & Rubiaceae & Mussaenda erythrophylla & Shrub \\
\hline Musanda (red) & Rubiaceae & Mussaenda erythrophylla rosea & Shrub \\
\hline Kamini & Rutaceae & Murrnaya paniculata & Tree \\
\hline Hasnahena & Solanaceae & Cestrum nocturumn & Tree \\
\hline Duranta & Verbenaceae & Duranta repens & Shrub \\
\hline Vat & Verbenaceae & Clerodendrom infortumatum & Shrub \\
\hline
\end{tabular}

Table 6. Biodiversity of aquatic plants observed in the area of Ramsagar

\begin{tabular}{|l|l|l|l||}
\hline \multicolumn{1}{|c|}{ Local name } & \multicolumn{1}{c|}{ Family } & \multicolumn{1}{c||}{ Genus Species } & Hant type \\
\hline \hline Kachuripana & Pontederiaceae & Eichhornia crassipes \\
\hline \hline Panikachu & Pontederiaceae & Monochoria hastate & Herb \\
\hline \hline Khudipana & Lemnaceae & Lemna minor & Herb \\
\hline \hline Topapana & Araceae & Pistia stratiotes & Herb \\
\hline \hline Kachu & Araceae & Colocasia esculenta & Herb \\
\hline \hline Sada Shapla & Nymphaceae & Nymphaea pubesceae & Herb \\
\hline \hline Panilong & Onagraceae & Ludwigia hyssopifolia & Herb \\
\hline \hline Helencha & Onagraceae & Jussleua repens & Herb \\
\hline \hline Kalmilata & Convolvulaceae & Ipomoea aquatic & Shrub \\
\hline \hline Dholkalmi & Convolvulaceae & Ipomoea fistulosa & Herb \\
\hline \hline Malancha & Amaranthaceae & Alternanthera philoxeroides & Herb \\
\hline \hline Jonia & Cyperaceae & Fimbristylis miliacea & Grass \\
\hline \hline Keshur & Cyperaceae & Cyperus michelianis & Grass \\
\hline \hline Dol & Gramineae & Hygrorhiza aristata & Herb \\
\hline \hline Arail & Gramineae & Leersia hexandra & Herb \\
\hline \hline Bishkatali & Polygonaceae & Polygonum hydropiper & Herb \\
\hline \hline Gangpalong & Polygonaceae & Rumex maritimus & Grass \\
\hline \hline Panimorich & Polygonaceae & Polygonum oriental & Herb \\
\hline
\end{tabular}

Table 7. Biodiversity of weed species observed in the study area

\begin{tabular}{|l||l||l||l||}
\hline \multicolumn{1}{|c|}{ Local name } & \multicolumn{1}{c|}{ Family } & \multicolumn{1}{c||}{ Genus Species } & \multicolumn{1}{c||}{ Plant type } \\
\hline \hline Shaknotey & Amaranthaceae & Amaranthus viridis & Herb \\
\hline \hline Shusni & Marseliaceae & Marsilia quadrifolia & Herb \\
\hline \hline Ghagra & Compositae & Xanthium italicum & Shrub \\
\hline \hline Bontula & Compositae & Sonchus arvensis & Herb \\
\hline \hline Shial mutra & Compositae & Blumea lacera & Herb \\
\hline \hline Mikania lota & Compositae & Mikania cordata & Herb \\
\hline \hline Bonkopi & Compositae & Gnaphalium affine & Herb \\
\hline
\end{tabular}




\begin{tabular}{|c|c|c|c|}
\hline Local name & Family & Genus Species & Plant type \\
\hline Bon sharisha & Cruciferae & Brassica kaber & Herb \\
\hline Bon mula & Cruciferae & Raphanus raphanistrum & Herb \\
\hline Bathua & Cheenopodiaceae & Chenopodium alabum & Herb \\
\hline Bhatshola & Leguminoceae & Aeschynomene aspera & Herb \\
\hline Katabegun & Solanaceae & Solanaum carolinense & Herb \\
\hline Tit begun & Solanaceae & Solanaum torytam & 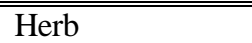 \\
\hline Foska begun & Solanaceae & Physalis heterophylla & Herb \\
\hline Kanaibashi & Commelinaceae & Cyanotis bengalensis & Herb \\
\hline Kanainala & Commelinaceae & Cyanotis axillaries & Herb \\
\hline Monayna & Commelinaceae & Commelia diffusa & Shrub \\
\hline Holde mutha & Cyperaceae & Cyperus esculenrus & Shrub \\
\hline Muthachaise & Cyperaceae & Fimbristylis diphylla & Shrub \\
\hline Panichaise & Cyperaceae & Eleocharis atroperpurea & Shrub \\
\hline Chechra & Cyperaceae & Seirpus mисronatus & Shrub \\
\hline Bara chucha & Cyperaceae & Cyperus iria & Shrub \\
\hline Sabuj nakful & Cyperaceae & Cyperus difformis & Shrub \\
\hline Khudi patai & Cyperaceae & Cyperus flavidus & Shrub \\
\hline Shakta khagra & Cyperaceae & Cyperus pilosud & Shrub \\
\hline Jonia & Cyperaceae & Cyperus miliacea & Shrub \\
\hline Chapragash & Gramineae & Elusine indica & Grass \\
\hline Anguligash & Gramineae & Digitaria sanguinalis & Grass \\
\hline Khudeyanguli & Gramineae & Digitaria ischaemum & Grass \\
\hline Sabujsialleza & Gramineae & Setaria viridis & Grass \\
\hline Haludshialleja & Gramineae & Setaria glarica & Grass \\
\hline Chiragash & Gramineae & Eragrostis gangetica & Grass \\
\hline Carpetgash & Gramineae & Axonopus compressus & Grass \\
\hline Premkata & Gramineae & Chrysopogon aciculatus & Grass \\
\hline Chlagash & Gramineae & Parapholis strigosa & Grass \\
\hline Monagash & Gramineae & Paspalum commersonii & Grass \\
\hline Ulugash & Gramineae & Imperata cylindrricas & Grass \\
\hline Durba & Gramineae & Cynodon dactylon & Grass \\
\hline Gitla gash & Gramineae & Paspalum distichum & Grass \\
\hline Chapatti gash & Gramineae & Paspalum conjagatum & Grass \\
\hline Khudeyshama & Gramineae & Echinochloa colonum & Grass \\
\hline Boro shama & Gramineae & Echinochloa crusgalli & Grass \\
\hline Fulka gash & Gramineae & Leptochloa chinesis & Grass \\
\hline Kakpaya & Gramineae & Dactyloclenium aegyptium & Grass \\
\hline Ban palong & Polygonaceae & Rumex maritimus & Herb \\
\hline Holdenakphul & Campanlaceae & Wahlenbergia marginata & Herb \\
\hline Tripatrishak & Leguminoceae & Desmodium triflorum & Herb \\
\hline Araich & Leguminoceae & Cassia tora & Herb \\
\hline Banmosur & Leguminoceae & Vicia savita & Herb \\
\hline Masurchana & Leguminoceae & Vicia hirsute & Herb \\
\hline Shalukdhekis & Nymphaeaceae & Nymphaea rubra & Aquatic \\
\hline Hak & Dryopteidaceae & Dryopteri serrate-dentata & Herb \\
\hline Nunia & Portulaceae & Portulaca oleracea & Herb \\
\hline Hazardana & Euphorbiaceae & Croton sparsiflorus & Herb \\
\hline Chotodudhia & Euphorbiaceae & Euphorbia hirta & Herb \\
\hline Borodudhia & Euphorbiaceae & Euphorbia sparsiflorus & Herb \\
\hline
\end{tabular}


According to the total available number of plant species by type; the weeds were the largest group having 57 species $(36.76 \%)$, the second largest group was the medicinal plants having 75 species $(27.57 \%)$ and the lowest group was the Palm plants having only 5 species $(1.84 \%)$ (Figure 1).

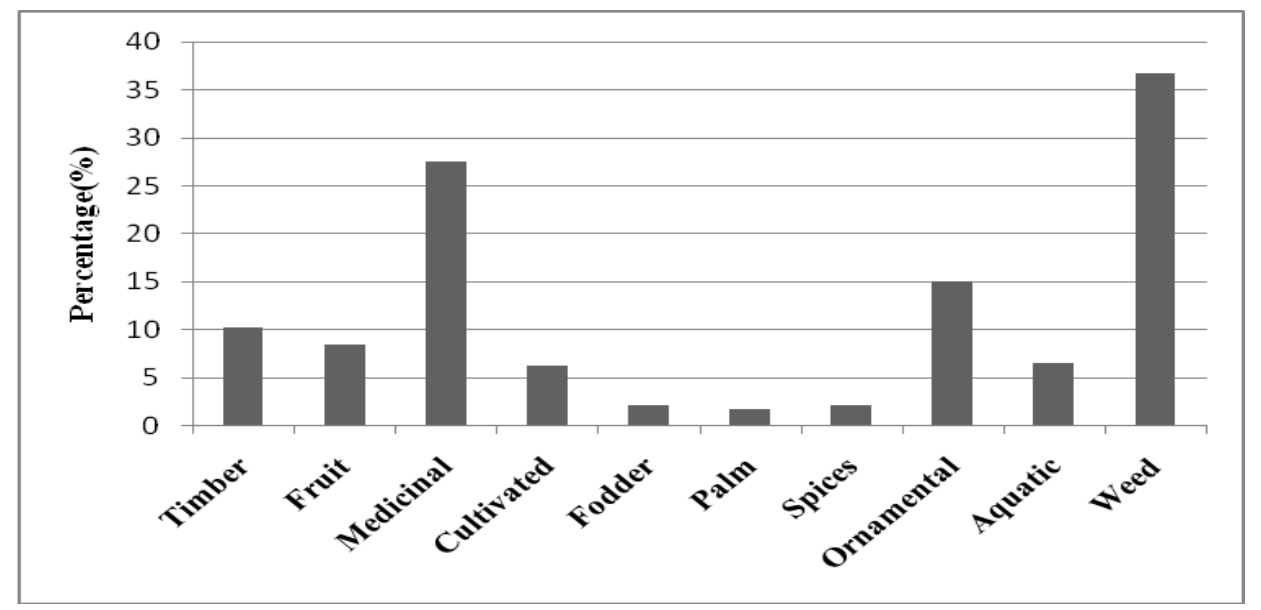

Fig 1. Percentage of total species for different groups of plants in the study area

\section{Animal Biodiversity}

Only one species among the cultivated fishes was found under the family Chicklidae which was Telapia (Oreochromis mossumbicas), the other 8 of cultivated fishes were found under the family Cyprinidae which included Mirror Carp (Cyprinus carpo), Silver Carp (Hypophthalmichthys molitfix), Grass Carp (Ctenopharyngodon idella), Catla (Catla catla), Rui (Labeo rohita), Mrigel (Cirrhinus mrigala), Sharputi (Puntius sarana), Kalbaush (Labeo calbasu). One species of cultivated fishes was found under the family Pangus Siluridae (Pangasius, pangasius) and another species found under family Cichilidae which included Nilotica (Oreochromis niloticus).

The diversity of indigenous fish species found in the study area included the following groups Needle fish climbing perch, Mud perch, Snake head, Goby, Glass fish, Catfish, Puffer, Loach, Spiny eel, Minnows and minors, Feather backs, Croaker, Carp, and Other as shown in the table 8 . There were some threatened small indigenous fish species in the study area which are liated in the table 9.

Table 8. Biodiversity of indigenous fish species found in the study area

\begin{tabular}{|c|c|c|c|}
\hline Group & Local name & Family & Genus Species \\
\hline \multirow[t]{3}{*}{ Needle fish climbing perch } & Kakila & Belonidae & Xenetodon cancila \\
\hline & Koi & Anabantidea & Anabas testudineus \\
\hline & Kholisha & Belontiidae & Colisa fasciatus \\
\hline \multirow{3}{*}{$\begin{array}{l}\text { Mud } \\
\text { perch }\end{array}$} & Napit koi & Nandidae & Badis badis \\
\hline & Bheda & Nandidae & Nandus nandus \\
\hline & Kuicha & Mastacembelidae & Monopterus cuchia \\
\hline \multirow[t]{4}{*}{ Snake head } & Taki & Channidae & Channa punctata \\
\hline & Shol & Channidae & Channa striata \\
\hline & Cheng & Channidae & Channa oriatalis \\
\hline & Gojar & Channidae & Channa marulius \\
\hline Goby & Bailla & Gobiidae & Glossogobius giuris \\
\hline Glass fish & Chanda & Ambassidae & Chanda nama \\
\hline \multirow[t]{5}{*}{ Catfish } & Magur & Clariidae & Clarius batrachus \\
\hline & Shing & Heteropneudtidae & Heteropneustes tossilis \\
\hline & Pabda & Siluridae & Ompok bimaculatus \\
\hline & Air & Bagridae & Aorichthys aor \\
\hline & Tengra & Bagridae & Mystus vittatus \\
\hline
\end{tabular}




\begin{tabular}{|c|c|c|c|}
\hline Group & Local name & Family & $\begin{array}{l}\text { Genus Species } \\
\end{array}$ \\
\hline & Batashi & Schibeidae & Pseuaeytropius atherinoides \\
\hline & Banspata & Schibeidae & Ailia punctata \\
\hline & Bacha & & Eutropiichthys vacha \\
\hline & Kazoli & Schibeidae & Ailia coila \\
\hline & Golsha & Bagridae & Myzus cavasius \\
\hline Puffer & Potka & Tetradontidae & Tetraodon cutcutta \\
\hline \multirow[t]{3}{*}{ Loach } & Rani & Cobitidae & Botia dario \\
\hline & Gutum & Cobitidae & Lepidocephalus guntea \\
\hline & Paharigutum & Cobitidae & Somileptes gongota \\
\hline \multirow[t]{3}{*}{ Spiny eel } & Sal baim & Mastacembelidae & Mastacembelus armatus \\
\hline & "Guchi & Mastacembelidae & Mastacembelus pancalus \\
\hline & Tara baim & Mastacembelidae & Mastacembelus aculeat \\
\hline \multirow[t]{8}{*}{ Minnows and minors } & Puti & Cypinidae & Puntius chola \\
\hline & Elang & Cypinidae & Rasbora elang \\
\hline & Mola & Cypinidae & Amblypharyngodon mola \\
\hline & Chela & Cypinidae & Salmostoma bacaila \\
\hline & Kash khaiya & Cypinidae & Chela laubuca \\
\hline & Dhela & Cypinidae & Osteobrama cotio \\
\hline & Tit puti & Cypinidae & Puntius tieoto \\
\hline & Jat puti & Cypinidae & Puntius sophore \\
\hline \multirow{2}{*}{$\begin{array}{l}\text { Feather } \\
\text { backs }\end{array}$} & Foli & Notopteridae & Notopterus notopterus \\
\hline & Chital & Notopteridae & Chitala chitala \\
\hline Croaker & Poa & Sciaenidae & Macrospinosa cuja \\
\hline \multirow{2}{*}{ Carp } & Nandil & Cyprinidae & Labeo nandina \\
\hline & Mahashol & Cyprinidae & Tor tor \\
\hline \multirow[t]{11}{*}{ Other } & Bamosh & Anguillidae & Ophisternon bengalense \\
\hline & Darkina & Cyprinidae & Rasbora rasbora \\
\hline & Boal & $\begin{array}{l}\text { Siluridae } \\
\end{array}$ & Wallagu attu \\
\hline & Khakila & & Rhinomugil corsula \\
\hline & Bhangra & Cyprinidae & Labeo boga \\
\hline & Chapila & Clupidae & Gudusia chapra \\
\hline & Chep chela & Cyprinidae & Danio devario \\
\hline & Ekthota & & "Dermogenys pusilla \\
\hline & Rita & & Rita rita \\
\hline & Piali & & Aspidoparia morar \\
\hline & Neptani & Belonatiidae & Ctenops nobilis \\
\hline
\end{tabular}

Table 9. Biodiversity of threatened Small Indigenous Fish Species (SIS) in the study area

\begin{tabular}{|l|l|l||}
\hline \multicolumn{1}{|c|}{ Local name } & \multicolumn{1}{c|}{ Gamily } & \multicolumn{1}{c||}{ Genus Species } \\
\hline \hline Darkina & Cyprinidae & Rasbora rasbora \\
\hline \hline Pabda & Siluridae & Ompok bimaculatus \\
\hline \hline Rani & Cobitidae & Botia dario \\
\hline \hline Tengra & Bagridae & Mystus vittatus \\
\hline \hline Sal baim & Mastacembelidae & Mastacembelus armatus \\
\hline \hline Bheda & Nandidae & Nandus nandus \\
\hline \hline Gol chanda & Ambassidae & Parambassis ranga \\
\hline \hline Dhela & Cypinidae & Osteobrama cotio \\
\hline \hline Kash khaiya & Cypinidae & Chela laubuca \\
\hline \hline Chela & Cypinidae & Salmostoma bacaila \\
\hline \hline Shol & Channidae & Channa striata \\
\hline
\end{tabular}




\begin{tabular}{|l|l|l||}
\hline \multicolumn{1}{|c|}{ Local name } & \multicolumn{1}{c|}{ Family } & \multicolumn{1}{c||}{ Genus Species } \\
\hline \hline Kakila & Belonidae & Xenetodon cancila \\
\hline \hline Tit puti & Cypinidae & Puntius tieoto \\
\hline \hline Jat puti & Cypinidae & Puntius sophore \\
\hline \hline Gojar & Channidae & Channa marulius \\
\hline \hline Mahashol & Cyprinidae & Tor tor \\
\hline \hline Magur & Clariidae & Clarius batrachus \\
\hline \hline Shing & Heteropneudtidae & Heteropneustes tossilis \\
\hline \hline Boal & Siluridae & Wallagu attu \\
\hline \hline Bhangra & Cyprinidae & Labeo boga \\
\hline \hline
\end{tabular}

Indigenous fish species are decreasing day by day. Causes behind decreasing indigenous fish species mainly attributed to over population, which causes over exploitation due to their poverty. Furthermore, agricultural pollution into the water bodies through rainfall and flood washout, destruction of breeding grounds of indigenous fish species due to construction, various diseases of fish were other major causes behind decreasing indigenous fish species.

The bank birds named Common Mayna (Acridotheres tristis), Bulbul (Pcynonotas jocosus) and Sociable Lapwing (Vanellus gregarious), observed in study area were resident and very common and common respectively in status. Other bank birds named Magpie Robin (Copsychus saularis), Dove (Streptopelia decaocto), Woodpecker (Chrysocolaptes lucidus), Weaver (Ploceus benghalesis) observed in RNP were few in status. Two bank birds - Common Starling (Sturnus vulgaris) and Sky Lark (Alauda arvensis) were migratory in status. The prey birds observed at the study area included Owl (Ketupa zeylonensis), Kite
(Haliastur Indus), Osprey (Pandion haliaetus), and Falcon (Falcon peregrines).

Among them owl was resident and few in status, while, the remaining three species were very few. The waterfowl named White Stork (Ciconia boyciana), Kingfisher (Alcedo atthis), Black Heron (Egretta ardesiaca) and Little Grebe (Tachybaptus ruficollis) observed in study area were resident and few in status. Grey Heron (Ardea cinerea), Pond Heron (Ardeola grayii), and Great Egret (Egretta alba), were common. Other waterfowl named Diver (Gavial stellata) were migratory and few, Night Heron (Nycticorax nycticorax) were threatened, Greylag Goose (Anser anser) were migratory and very few and Duck (Cairina scutulata) were domestic and common.

Table 10. shows the biodiversity of miscellaneous faunal species found in the study area with their status. Most of them were found to be common, three were threatened (Jackal, Weasel and Sona Toad) and three were few (Small Prawn, Leech and Kobra) while only one was very few (Guisap).

Table 10. Biodiversity of miscellaneous faunal species found in the study area

\begin{tabular}{|l||l||l||}
\hline \multicolumn{1}{|c|}{ Common name } & \multicolumn{1}{c|}{ Genus Species } & \multicolumn{1}{|c||}{ Status } \\
\hline \hline Freshwater Mussel & Lamellidens marginalis & C \\
\hline \hline Apple Snail & Pila globosa & C \\
\hline \hline Freshwater Crab & Paratel masoniana & C \\
\hline \hline Mud Crab & Scylla serrata & C \\
\hline \hline Small Prawn & Palaemon sp. & F \\
\hline \hline Leech & Hirudinaria granulose & F \\
\hline \hline Jackal & Vulpes bengalensis & WT \\
\hline \hline Weasel & Herpestes urva & WT \\
\hline \hline Snake & Cerberus rhynchops & C \\
\hline \hline Guisap & Varanus bengalensis & VF \\
\hline \hline Kobra & Naja naja & F \\
\hline \hline Sona Toad & Rana tigrina & T \\
\hline \hline Common Toad & Bufo melanostictus & C \\
\hline \hline Rat & Ratus bengalensis & C \\
\hline
\end{tabular}

C: Common, F: Few, VF: Very Few, T: Threatened, W: Wild 
Total 73 species of fishes were observed in the study area. Indigenous fish was the largest group having 53 species $(63.10 \%)$, then the threatened small indigenous fish was 20 species $(23.81 \%)$, and cultivated fish group contained 11 species (13.10\%). Birds were recorded into three groups named Dank birds, Bird of pryaud waterfoud and the percentage of these group are showd in (Figure 2).

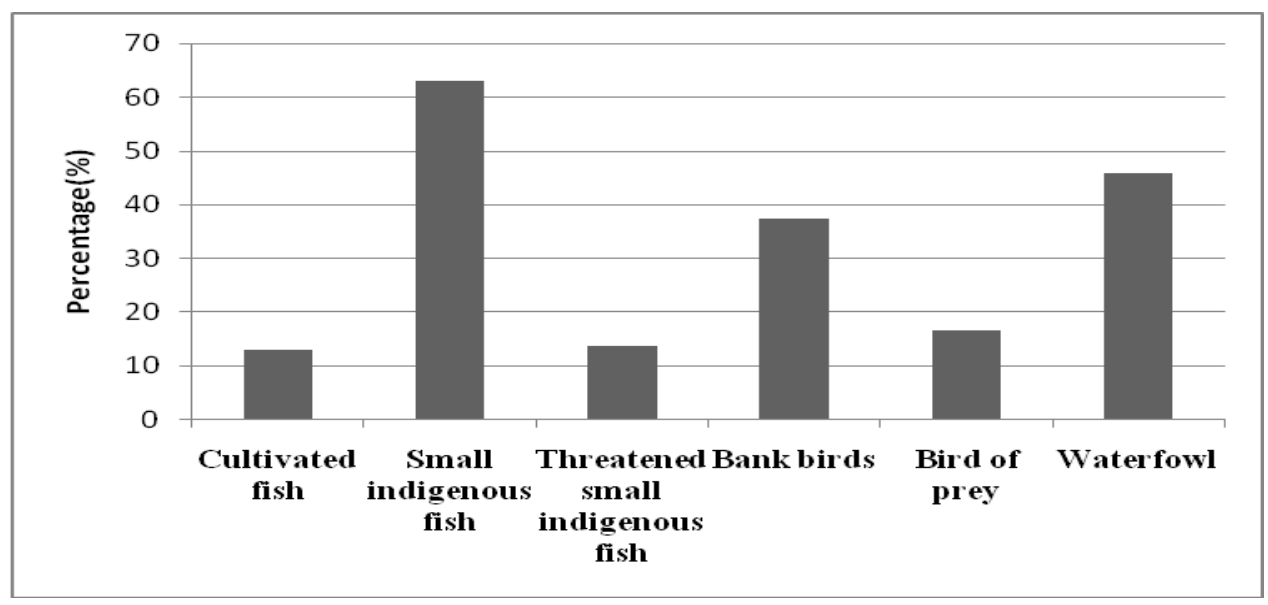

Figure 2. Percentage of total species for different groups of fauna in the study area

\section{Contribution of biodiversity to local people}

The contribution of floral and faunal biodiversity to the local people in the study area was mainly for food. Although medicinal plants were used for medicine purpose, weeds and fodder plants were used as a food for domestic animals, timber plants were used for making furniture or as fuel wood etc.

\section{Management of RNP}

There was only 8 staff for maintaining the National Park. There was no management plan in the RNP but they had annual work plan. There was boundary constructed around the National Park.

Table 11. Overall management of RNP

\begin{tabular}{|l|c|}
\hline Management Plan & No. \\
\hline Annual Work Plan & Yes. \\
\hline Boundary Demarcation & Yes and boundary wall is constructed. \\
\hline Management Zoning & No. \\
\hline Inventory & Not applicable. \\
\hline Land use & Indigenous plantations-18.22 ha, Exotic plantations-2 ha. \\
\hline Roads and Bridges & Roads and foot trails have been developed. \\
\hline Miscellaneous works & Provisions construct picnic sheds and cottages. \\
\hline
\end{tabular}

There were only 8 staffs including 1 forest officer, 3 gardeners, 3 guards and 1 watchman in the RNP for maintaining the management system which cannot be adequate for proper management of this Park.

\section{Conclusion}

A heterogeneous assemblage of 367 species including 256 plant species, 24 species of waterfowl and birds, 14 species of miscellaneous fauna and 73 species of fish could be recorded at the study area. Contribution of biodiversity to the local people was mainly for food, although they used plant species as wood for making furniture, fuel wood, medicinal purpose, etc. The main cause of decreasing indigenous fish species was over exploitation of fish due to over population, other causes were agricultural pollution, diseases, flood, etc. There was inadequate staff for maintaining the National Park. This clearly indicated the need for more attention from the Government to take practical endeavors for proper management of the Park and thereby conserve its biodiversity. 


\section{References}

Banglapedia. 2006. National Encyclopedia of Bangladesh. Asiatic Society of Bangladesh.

HUDA, K. S. 2008. Co-management of protected areas in Bangladesh: A strategy for establishing an institutional framework. Nishorgo Support Project, Bangladesh Forest Department (BFD), Ministry of Environment and Forest, Government of Bangladesh. Availableathttp://www.nishorgo.org/referenc e_center.asp.

IRG (International Resources Group). 2004. Assessment of the Forest Department's institutional organization and capacity to manage the protected area system of Bangladesh. Nishorgo Support Project Contract No. 388-c-00-03-00050-00, International Resources Group (IRG), 1211 Connecticut Avenue, Washington DC 20036, USA.
Islam, M. S. 2006. Biodiversity of Jamuna Bridge of the surrounding area, M.S. thesis, Department of ENVS, BAU, Maymensingh, Bangladesh. Page No. 9-15.

Islam, S. 2001. The status of Bhawal National Park: are the objectives of protected areas being met? Technical Report, Independent University, Bangladesh.

Rahman, A. K. A. 1989. Fresh Water Fisheries of Bangladesh. Bangladesh Zoological Society, Department of Fisheries, Dhaka, Bangladesh. Page No. 364.

Rashid, M. 1990. Fuler Chash Bangla Academy, Dhaka, Bangladesh. Page No. 34-172 